\title{
Physical properties of gastrointestinal stromal tumors based on atomic force microscope analysis
}

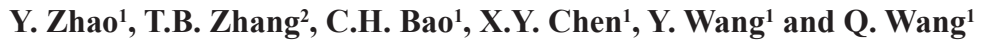 \\ ${ }^{1}$ Department of General Surgery, \\ Shengjing Hospital of China Medical University, \\ Shenyang, Liaoning Province, China \\ ${ }^{2}$ Department of Biochemistry and Molecular Biology, \\ China Medical University, Shenyang, Liaoning Province, China \\ Corresponding author: Q. Wang \\ E-mail: wangqiangme@yeah.net
}

Genet. Mol. Res. 12 (4): 5774-5785 (2013)

Received February 26, 2013

Accepted July 29, 2013

Published November 22, 2013

DOI http://dx.doi.org/10.4238/2013.November.22.4

\begin{abstract}
This study was designed to detect the stiffness of single living gastrointestinal stromal tumor (GIST) cells in vitro using an atomic force microscope as a probe tool. We determined that the stiffness of living GIST cells was $3913 \mathrm{~Pa}$, the stiffness of the membrane was $642 \mathrm{~Pa}$, and the stiffness of the cytoplasm was $17,550 \mathrm{~Pa}$. For comparison, we also determined the stiffness of a normal stomach cell, which was $7374 \mathrm{~Pa}$, and that of in vitro GIST cells after $2 \mathrm{~h}$ of exposure, which was 10,680 Pa. Measuring the mechanical properties of individual GIST cells might provide more complementary information for the diagnosis and treatment of GISTs from the perspective of physical characteristics.
\end{abstract}

Key word: Physical properties; Gastrointestinal stromal tumors; Atomic force microscope 


\section{INTRODUCTION}

Gastrointestinal stromal tumors (GISTs) are mesenchymal tumors that have only been observed within the last few decades (Hirota et al., 1998; Al-Jehani et al., 2011; Grotz and Donohue, 2011; Miettinen and Lasota, 2011; Mitchell et al., 2011; Bamboat and DeMatteo, 2012; Coccolini et al., 2012; Grover et al., 2012; Vazquez et al., 2012). There is no significant difference among GIST patients of different ethnicities or between genders. GISTs can occur in any tissue of the digestive tract. The most common primary GIST sites are the stomach and small intestine, followed by the rectum and the gullet. GISTs can also develop in the omentum, retroperitoneal mesangial, and parenteral tract. The development of GISTs involves several types of cellular activities and physiological processes, although malfunction of cellular signal transmissions is the major contributing factor. To date, most GISTs have been associated with activating mutations in particular genes, including the KIT or homologous KIT receptor tyrosine kinase, platelet-derived growth factor receptor alpha, and the phosphatidylinositol 3-kinase genes (Subramanian et al., 2004; Sihto et al., 2005; Hennessy et al., 2005; Gomes et al., 2007; Daniels et al., 2011; Jaramillo et al., 2012; Wang et al., 2012). These gene mutations play important roles in cancer generation, development, metastasis, and treatment. With the emergence of the target therapy medicine imatinib (Eisenberg and Judson, 2004; Pisters and Colombo, 2011; Joensuu et al., 2012; Li et al., 2012; Miranda et al., 2012), the response and recovery of GIST patients has been greatly improved. However, in a subset of cases, the tumor remains unstable under imatinib treatment, and in a minority of cases, tumor regrowth is observed (Heinrich et al., 2006).

Results of biological experiments have long been deduced from data analysis methods based on measurements of large ensembles of cells (Lipman et al., 2003; Jaiswal and Simon, 2007). In this way, macro phenomena of biological activities can be directly observed or chemically recorded. However, individual cell variations are masked by ensemble measurements. In order to reveal the mechanism of cell physiological activities in more detail, atomic force microscope (AFM) (Binning et al., 1986) analysis technology is a reasonable method to complement conventional ensemble measurements for obtaining information at the single cell level (Charras and Horton, 2002; Cuerrier et al., 2007; Helenius et al., 2008).

In this study, we investigated the elastic properties of GISTs using AFM. These properties included the stiffness of living GISTs, the GIST membrane, and the GIST skeleton. Furthermore, the stiffness of normal stomach cells was detected for comparison. Differences between GIST and normal cells can be used as a biomarker to aid discovery in developing effective treatments for GISTs (Edwardson and Henderson, 2004). In addition, we detected the stiffness of GIST cells $2 \mathrm{~h}$ after exposure to the room environment.

\section{MATERIAL AND METHODS}

The GIST specimens used in this study were donated by patients in our department (Gastrointestinal Surgery of Shengjing Hospital Affiliated to China Medical University). Our department has been engaged in basic and clinical research related to gastrointestinal tumors for several years. Every year, we perform approximately 250 operations related to gastrointestinal tumors, and GISTs comprise approximately 50 of these cases.

All patients signed written informed consent forms, and this study was supported by 
the Ethics Committee of Shengjing Hospital Affiliated to China Medical University. In our department, the tissues extracted from patients must meet the following conditions before being diagnosed as GISTs: the morphology of the tumor is consistent with typical morphology of GISTs and immunohistochemistry is CD117 (+), or the morphology of the tumor is consistent with that of typical GISTs and is CD117 (-), but is CD34 (+) or CD117 (-), CD34 (-), SMA (-), Desmin (-), and S-100 (-), and there is no indication of smooth muscle-derived or neurogenic tumors. Figure 1 shows a gross GIST specimen from a 25-year-old male patient.

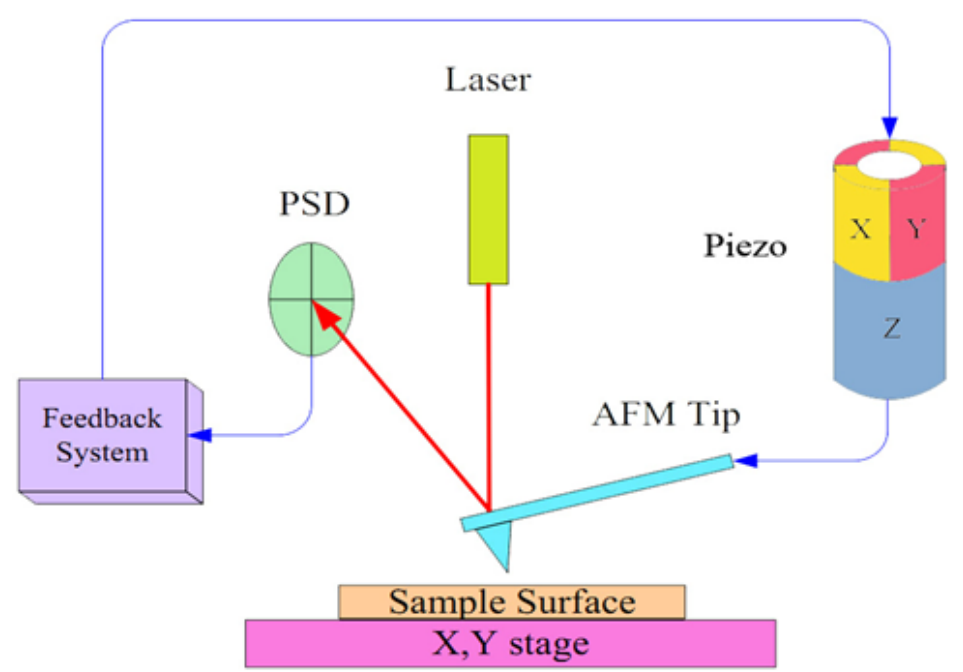

Figure 1. Atomic force microscope (AFM) principle. The deflection signal of the cantilever is detected by a laser beam focused on the free end of the cantilever and then reflected into a photodiode signal detector (PSD). The PSD signal is amplified by the lock-in amplifier and then passed to the feedback circuit. The feedback circuit processes the PSD signal according to the setpoint value, and then delivers the Z piezo up and down according to the sample morphology.

\section{Sample preparation}

The GIST tumor tissue was processed immediately after the surgery. All procedures were carried out in a biological safety cabinet (Thermo Fisher Scientific) using sterile tools. The tissue was first immersed in 75\% ethanol (Sigma-Aldrich) for $10 \mathrm{~min}$, and then the external epidermis was removed, leaving only a small piece of tissue in the center. This small piece was placed on a Petri dish and ground into powder. Then, $5 \mathrm{~mL} 0.25 \%$ trypsin (Invitrogen) was added to the Petri dish and the tissue was cut with small scissors for 1 min. The Petri dish was covered and transferred to the $\mathrm{CO}_{2}$ cell culture incubator (Thermo Fisher Scientific) at $37^{\circ} \mathrm{C}$ and $5 \% \mathrm{CO}_{2}$. The digest was terminated after $10 \mathrm{~min}$ with the addition of $10 \mathrm{~mL}$ culture medium (RPMI 1640, Thermo) with 10\% fetal bovine serum (Invitrogen) and 1\% penicillin and streptomycin (Invitrogen), and the mixture solution was filtered with a filter griddle. The filtered mixture was then centrifuged for $3 \mathrm{~min}$ at $100 \mathrm{~g}$ twice, and the supernatant was removed. After the first centrifugation, cells were suspended in culture medium, whereas after the second centrifugation, cells were suspended in phosphate-buffered saline (PBS). 
In order to determine the stiffness of the cells based on force curves, the cells must first be attached to the glass slide. To accomplish this, we used $0.1 \mathrm{mM}$ poly-L-lysine (SigmaAldrich) to modify the surface of the slide, which was then incubated overnight. Two hundred to $300 \mu \mathrm{L}$ GIST solution was then absorbed to the modified slide. The GISTs were fixed well on the slide 10 min later, and the slide was immersed onto a Petri dish that was filled with PBS.

\section{AFM measurement}

The AFM works in a raster fashion by scanning a very small tip mounted at the end of a flexible silicon micromachining cantilever that is in gentle contact with the sample surface, as shown in Figure 2. The interaction force between the tip and the sample is monitored by the deflection of the soft cantilever. The AFM can work with aqueous matter under physiological conditions, thereby enabling observations of cell activities in vitro (Doktycz et al., 2003). In particular, AFM can also be used as an actuator and not only as a detector. For example, AFM can locally measure the elastic properties of materials such as cells (Radmacher, 1997).

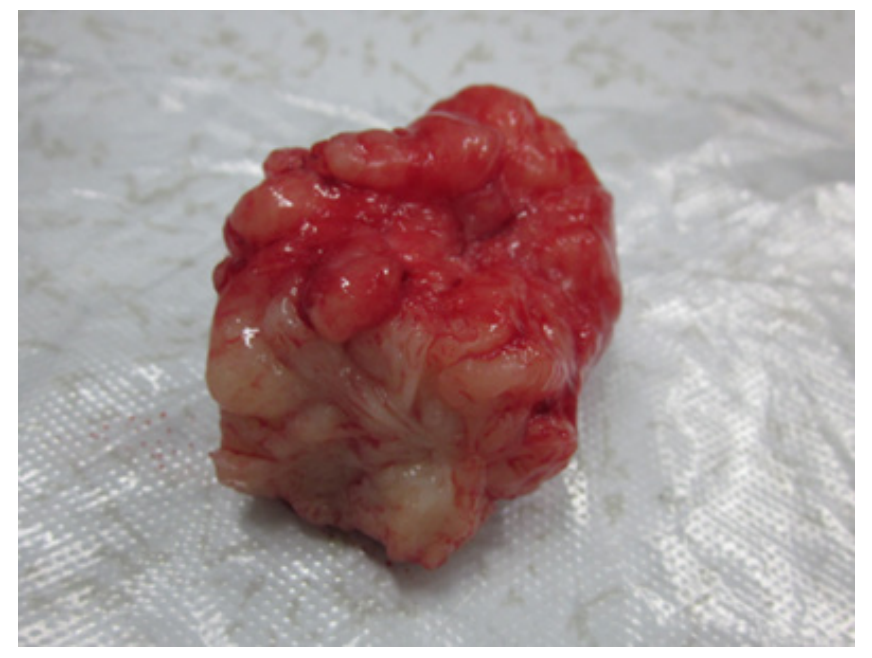

Figure 2. Gastrointestinal stromal tumor specimen. The diameter of this tumor is about $5.5 \mathrm{~cm}$ and is located in the fundus of the stomach, convex to gastric cavity.

The AFM can be used in many modes such as scanning, manipulating, and force ramping (Dufrêne, 2002). In order to obtain the stiffness parameter, the AFM works as an "indentor" to touch the object surface and then depart to form a force curve. Every single force curve consists of an approach-retract cycle, as shown in Figure 3 where the blue line represents the AFM tip approach to the object, while the red line represents retraction of the AFM tip from the object surface. If the stiffness of the sample is very soft, the tip will slightly deform when it touches the surface of the sample. In such cases, a smooth force curve will be obtained, as shown in Figure 3b, and can be used to calculate the Young's modulus (a measure of stiffness), which is also very soft. Conversely, a hard Young's modulus force curve can also be obtained, as shown in Figure 3a. 
a

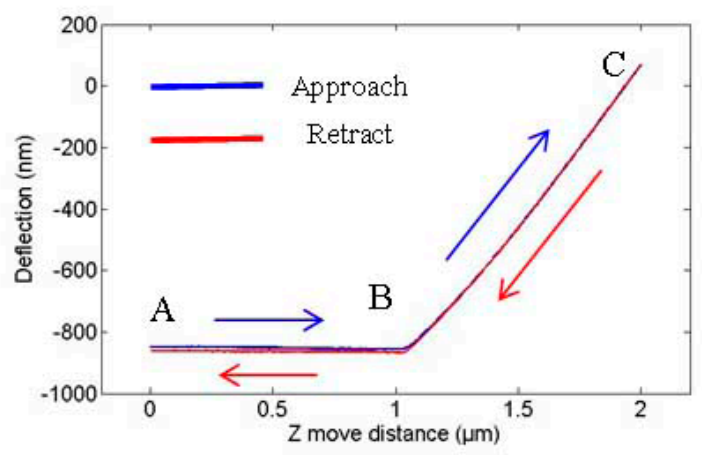

b

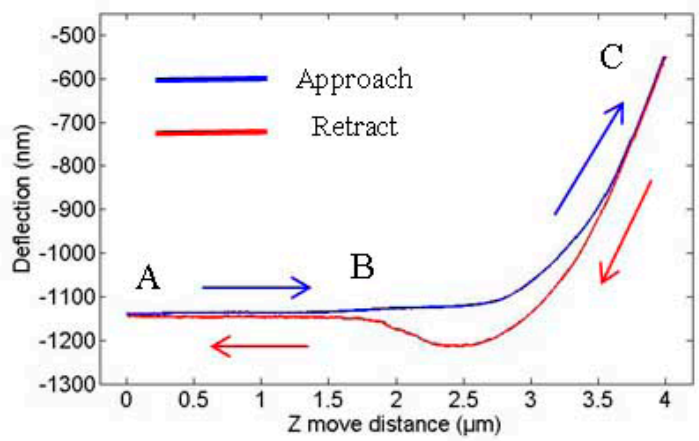

Figure 3. Force curves of the atomic force microscope (AFM). Point $A$ is the starting movement position of the $\mathrm{Z}$ piezo; point $B$ is the position that the AFM tip touches the sample; point $C$ is the ending movement position of the $\mathrm{Z}$ piezo. The blue line represents the approach sample process, while the red line goes the opposite direction. $\mathbf{a}$. Doing force curve on a hard surface. This is also the tip sensitivity calibration procedure. b. Doing force curve on cells. The approach line and the retract line are mismatched because the soft cell deforms a lot under indenting and also some deformation is plastic and cannot recover when indenting force is away.

The experiments were carried out with the Nanoscope IVa AFM system (Digital Instruments Inc., Santa Barbara, CA, USA) and the commercial Nanoscope software. The extremely soft MLCT AFM tip was used, which was suitable for detecting the very soft cell membrane. The experiments were performed at room temperature in PBS solution.

\section{RESULTS}

We detected the stiffness of living GISTs and also designed two comparative experiments. The first compared stiffness between living normal cells and GISTs, and the other compared variations in GIST stiffness over time, by comparing stiffness levels just after sample preparation and $2 \mathrm{~h}$ later in vitro. In addition, we determined the difference in stiffness between the membrane and the cytoplasm of living GISTs in vitro.

\section{Stiffness of living GISTs}

In this experiment, we indented the living cells in vitro just after the preparation. Each 
cell showed 25 force curves in a 5 x 5 matrix with a separation distance of $200 \mathrm{~nm}$ during each indentation. We ensured that the indenting force on the living cell was no more than $1 \mathrm{nN}$. Figure 4 shows a representative example of 25 force curves from one cell. If the approach curves and retract curves are nearly the same, the 25 force curves were considered to be good data; otherwise, the data were excluded from further analysis. Such cases of unequal approach and retraction curves may be caused by indenting the edge of the cell or by indenting an unhealthy cell. In addition, such data might result from thermal draft of the laser reflection.

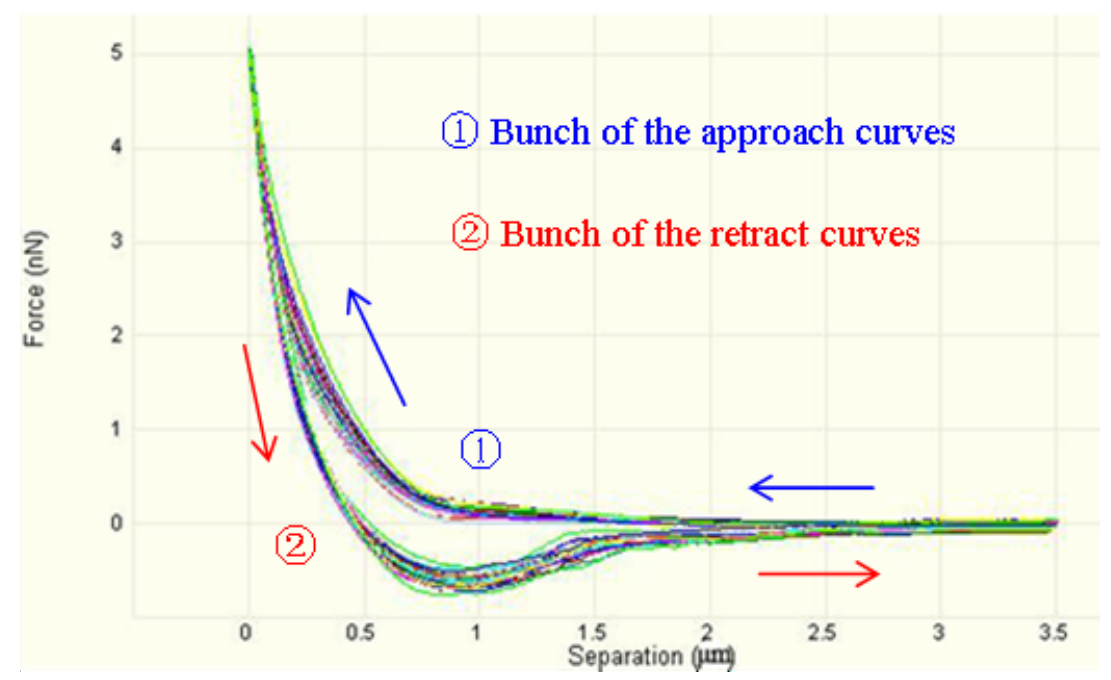

Figure 4. Bunch of coincidence force curves.

Figure 4 shows the profiles of the approach and retract curves that were used to calculate the stiffness of the cell. A simple model for calculating the stiffness of the cell is the Hertz model (Svaldo et al., 2006). The Hertz model can be applied to an extremely thick and soft sample. It also can be applied to thin films as long as the indentations in the sample by the tip are sufficiently small compared with its thickness (Rotsch et al., 1999). The following equation was used:

$$
\text { cone } \frac{2 \delta \tan \alpha}{\pi(1 \quad v)}
$$

where $F$ is the indenting force, $E$ is the Young's modulus, $\delta$ is the indenting depth, $\alpha$ is the radius of the tip end of the AFM $\left(\alpha=20^{\circ}\right)$, and $v$ is the Poisson ratio of the sample (here, $v=0.5$ ).

In order to calculate the $E$ more accurately, we used the approach curve only (the blue arrows in Figure 4) in the calculation, because the AFM tip might cause a few deformations during the retracting process. For the same reason, we never indented a second force curve in the same location.

The $\delta$ and the indenting force were obtained from the force curves, and then we de- 
veloped a MATLAB program to calculate the Young's modulus. Because 25 force curves were obtained from a single living cell, 25 different Young's modulus values can be determined. These moduli were clustered in a histogram and fit with a Gaussian curve as shown in the red profile of Figure 5A. The peak point of the curve showed that the stiffness of one selected living GIST cell was $3801 \mathrm{~Pa}$. As one cell has one fitting Young's modulus value, 100 living cells have 100 fitting $E$ values. The 100 fitting $E$ values were clustered in a histogram and fit with a Gaussian curve. The top center value of the Gaussian fitting profile was $3913 \mathrm{~Pa}$, which indicated that the stiffness of the GISTs in vitro was $3913 \mathrm{~Pa}$ (Figure 5B).
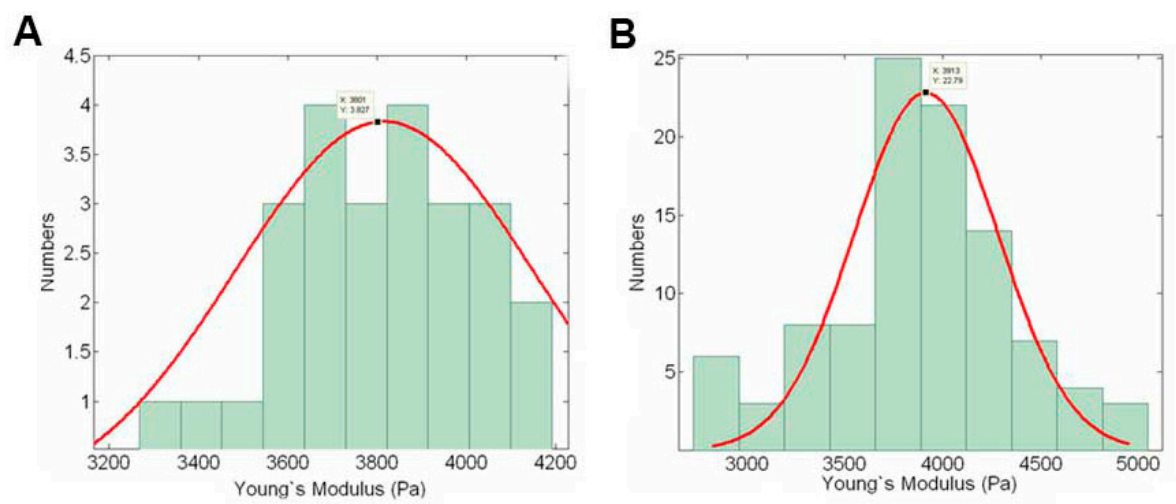

Figure 5. Stiffness of the living gastrointestinal stromal tumor cells in vitro. A. Process of getting the Young's modulus on one cell. B. Young's modulus fitting 100 cells.

The Young's modulus obtained through AFM represented the stiffness of the test point. A high value indicates a hard surface point, whereas a low value indicates a relatively soft surface point. The stiffness map of the cell was constructed by entering all of the force curves on the cell membrane into a matrix. Every matrix was 200 x $200 \mathrm{~nm}$. As shown in Figure 6, a 10 x 10-matrix force curve was constructed for the living cell, the Young's modulus of every point was calculated, and then the $E$ values were plotted in grayscale so that soft areas of the cell are colored light gray and harder areas are colored dark gray.

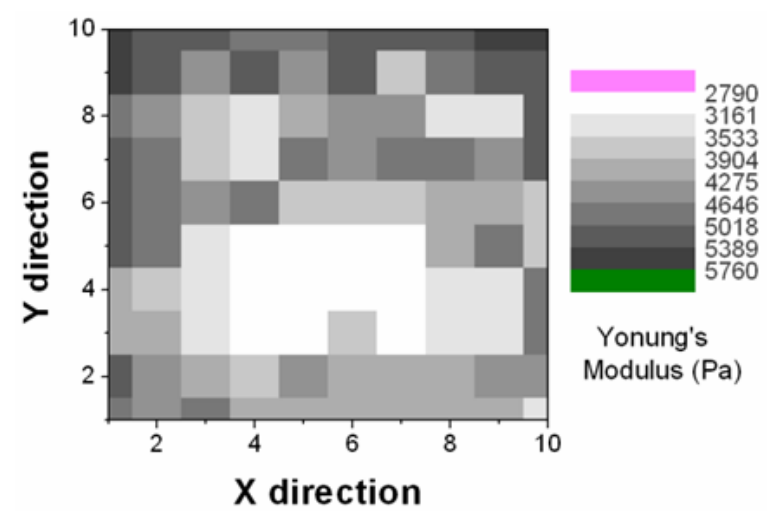

Figure 6. Mapping of the stiffness of the living gastrointestinal stromal tumor cells (in grayscale). The lighter color means the area of the softer cell. 
Close examination of the force curves obtained from the living GIST cells in vitro revealed an inflection point on every approaching force curve (Figure 7). The slope of deflection rises gently from the contact point to the inflection point, which suggests that the AFM tip indented the soft membrane only. As the AFM tip moved down past the inflection point, the slope rises steeply, which indicates that the AFM tip touched something stiffer than it did before. This sudden rising event may be caused by the organelles in the cell. We constructed a force curve in a $5 \times 5$ matrix and calculated the Young's modulus before and after the inflection point, which represents the stiffness of the membrane and the cytoplasm of the same cell, respectively. As shown in Figure 8, the membrane stiffness was $642 \mathrm{~Pa}$, and the cytoplasm stiffness was $17,550 \mathrm{~Pa}$, indicating that the membrane is much softer than the cytoplasm in the cell. Therefore, the Young's modulus of $3913 \mathrm{~Pa}$ that was detected in the living GIST cell reflects the stiffness of the membrane and the skeleton.

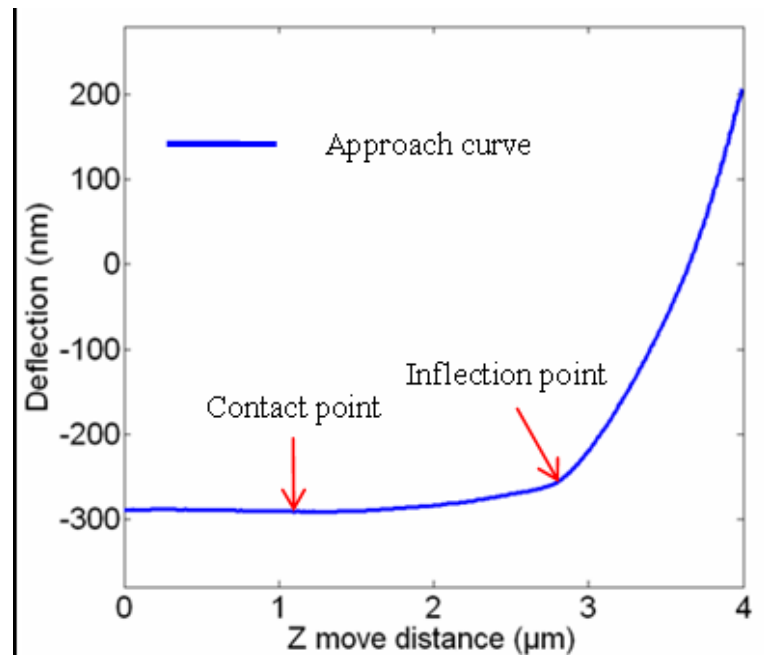

Figure 7. Approach curve obtained from gastrointestinal stromal tumor cells in vitro.
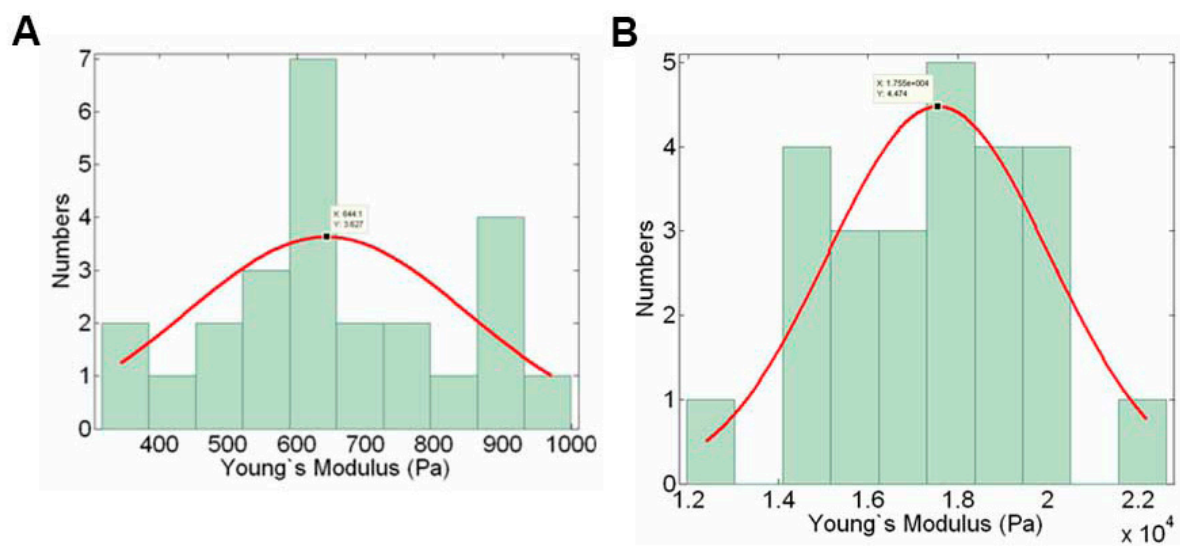

Figure 8. Decomposing of the gastrointestinal stromal tumor's stiffness. A. Stiffness of the membrane, which is about $642 \mathrm{~Pa}$. B. Stiffness of the cytoplasm, which is 17,550 Pa. 


\section{Stiffness comparison}

The previous experiment focused on stiffness of living GISTs. For comparison, we also measured the stiffness of normal cells and living GISTs in vitro $2 \mathrm{~h}$ after preparation, when the living cell may be in poor condition. For each cell type, we measured 100 cells, derived 5 x 5-matrix force curves for each cell, and calculated the Young's modulus for each force curve. The histogram of the 100 Young's moduli is shown in Figure 9. The stiffness of the normal stomach cell was found to be $7374 \mathrm{~Pa}$, which is slightly harder than that of the GISTs (3913 Pa), suggesting that normal stomach cells become softer when they develop into sick tumor cells. Two hours later, the stiffness of the GISTs in vitro was $10,680 \mathrm{~Pa}$, which indicates that the GISTs became harder over time until dying. The inflection point $2 \mathrm{~h}$ later was similar to that observed in fresh GIST cells in both cell types. We calculated the stiffness of the membrane and the skeleton of these two cell types using the same method as described above. Differences in stiffness among the three cell types are shown in Table 1. We could conclude that the membrane and the skeleton show the same characteristics as the whole cell. Additionally, regardless of cell type, the cell membrane was always much softer than the skeleton.
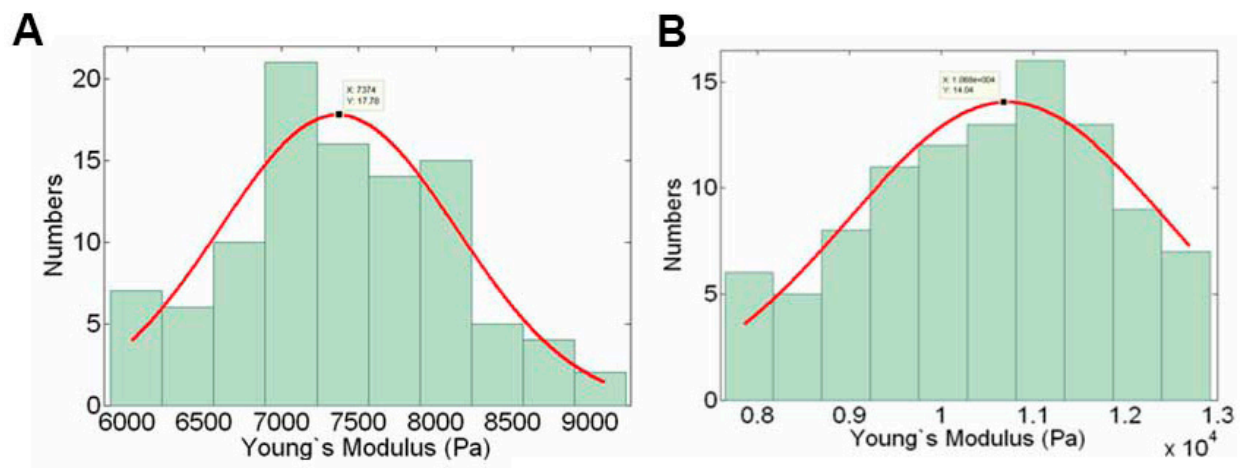

Figure 9. Stiffness comparison. A. Stiffness of the gastrointestinal stromal tumor cells $2 \mathrm{~h}$ later in vitro. B. Stiffness of the normal stomach cell.

Table 1. Stiffness comparison in gastrointestinal stromal tumor (GIST) cells.

\begin{tabular}{lccc}
\hline Stiffness $(\mathrm{Pa})$ & Normal cell & Living GISTs & GISTs 2 h later \\
\hline The whole cell & 7,374 & 3,913 & 10,680 \\
The membrane & 1,231 & 642 & 17,50 \\
The skeleton & 33,550 & 17,550 & 47,830 \\
\hline
\end{tabular}

\section{DISCUSSION}

The elastic property (stiffness) is one of the physical properties of cells (Bloom et al., 1991). Different types, shapes, or parts of cells can differ greatly with respect to stiffness. Stiffness might also vary in the same cell across differentiation periods. Any deviations from normal elastic properties of living cells may lead to diseases; however, diseases can also give rise to structural and elastic property changes (Li et al., 2008). Regardless of 
the causal direction, cell stiffness is closely associated with cell health status. No previous study has evaluated whether GISTs and normal stomach cells differ in stiffness. This is an important consideration for applications of the relationship between biomechanics and human diseases.

GIST invasion and metastasis is a complicated process involving interactions between GIST cells and normal cells. In some patients, the cancer cells transfer quickly, whereas other patients can survive for a few years. The rate of cancer cell transfer might be related to differences in the physical properties of cancer and normal cells. The results of the present study demonstrated that GIST cells are much softer than normal cells. Therefore, cell stiffness can be used as a clear biomarker, which might play a critical role in GIST treatment. Our ultimate goal is to investigate the spatial distribution of the mechanical properties of cellular structures from a single cell using AFM, with the aim of providing complementary methods to help treat, and potentially cure, GISTs.

In general, GIST-related research focuses on drug tests aiming to cure the disease. However, more comprehensive and in-depth knowledge from other scientific fields is greatly needed toward this goal. In this study, we evaluated GISTs from the perspective of their physical properties, and determined the stiffness of living GISTs. We further showed that the cytoplasm is much harder than the membrane. In addition, we found that GIST cells got harder over time, and that they are slightly softer than normal cells. These results strongly demonstrate that physical properties of cells could be used as effective biomarkers, which can play an important role in drug testing experiments. Currently, decisions related to diagnosis and targeted therapies for GIST mainly rely on immunohistochemical observations of paraffin section specimens of GIST; however, such specimens have lost their biological activity at the time of observation. AFM enables observations of the physical characteristics of a single living GIST cell under physiological conditions, allowing observations of real-time changes in the physical characteristics of living cells after targeted drug therapy, which can help to determine the sensitivity of tumor cells to drugs. Compared to immunohistochemical methods, AFM detection is fast, direct, and can operate under conditions close to the physiological state of cells. AFM can therefore be extremely effective for the direct diagnosis of GIST cells and for determining the sensitivity of targeted drug therapy. In addition, the stiffness of GISTs might be related to de-differentiation of the cells, which would suggest that a certain tumor grade could have a corresponding degree of stiffness. This relationship will be investigated in our future study.

\section{ACKNOWLEDGMENTS}

We would like to thank the Department of Gastrointestinal Surgery of Shengjing Hospital Affiliated to China Medical University for providing human GIST tissue specimens. We also thank the college of China Medical University for technical assistance in the experiments. Research supported by the Program Funded by Liaoning Province Education Administration (\#L2012295).

\section{Conflicts of interest}

The authors declare no conflict of interest. 


\section{REFERENCES}

Al-Jehani YM, Bousbait HI and Kant BR (2011). Extra-gastrointestinal stromal tumor presenting as a surgical emergency. Turk. J. Gastroenterol. 22: 617-620.

Bamboat ZM and DeMatteo RP (2012). Updates on the management of gastrointestinal stromal tumors. Surg. Oncol. Clin. N. Am. 21: 301-316.

Binnig G, Quate CF and Gerber C (1986). Atomic force microscope. Phys. Rev. Lett. 56: 930-933.

Bloom M, Evans E and Mouritsen OG (1991). Physical properties of the fluid lipid-bilayer component of cell membranes: a perspective. Q. Rev. Biophys. 24: 293-397.

Charras GT and Horton MA (2002). Single cell mechanotransduction and its modulation analyzed by atomic force microscope indentation. Biophys. J. 82: 2970-2981.

Coccolini F, Catena F, Ansaloni L and Pinna AD (2012). Gastrointestinal stromal tumor and mitosis, pay attention. World J. Gastroenterol. 18: 587-588.

Cuerrier CM, Lebel R and Grandbois M (2007). Single cell transfection using plasmid decorated AFM probes. Biochem. Biophys. Res. Commun. 355: 632-636.

Daniels M, Lurkin I, Pauli R, Erbstosser E, et al. (2011). Spectrum of KIT/PDGFRA/BRAF mutations and phosphatidylinositol-3-kinase pathway gene alterations in gastrointestinal stromal tumors (GIST). Cancer Lett. 312: 43-54.

Doktycz MJ, Sullivan CJ, Hoyt PR, Pelletier DA, et al. (2003). AFM imaging of bacteria in liquid media immobilized on gelatin coated mica surfaces. Ultramicroscopy 97: 209-216.

Dufrene YF (2002). Atomic force microscopy, a powerful tool in microbiology. J. Bacteriol. 184: 5205-5213.

Edwardson JM and Henderson RM (2004). Atomic force microscopy and drug discovery. Drug Discov. Today 9: 64-71.

Eisenberg BL and Judson I (2004). Surgery and imatinib in the management of GIST: emerging approaches to adjuvant and neoadjuvant therapy. Ann. Surg. Oncol. 11: 465-475.

Gomes AL, Bardales RH, Milanezi F, Reis RM, et al. (2007). Molecular analysis of c-Kit and PDGFRA in GISTs diagnosed by EUS. Am. J. Clin. Pathol. 127: 89-96.

Grotz TE and Donohue JH (2011). Surveillance strategies for gastrointestinal stromal tumors. J. Surg. Oncol. 104: 921-927.

Grover S, Ashley SW and Raut CP (2012). Small intestine gastrointestinal stromal tumors. Curr. Opin. Gastroenterol. 28: $113-123$.

Heinrich MC, Corless CL, Blanke CD, Demetri GD, et al. (2006). Molecular correlates of imatinib resistance in gastrointestinal stromal tumors. J. Clin. Oncol. 24: 4764-4774.

Helenius J, Heisenberg CP, Gaub HE and Muller DJ (2008). Single-cell force spectroscopy. J. Cell Sci. 121: 1785-1791.

Hennessy BT, Smith DL, Ram PT, Lu Y, et al. (2005). Exploiting the PI3K/AKT pathway for cancer drug discovery. Nat. Rev. Drug Discov. 4: 988-1004.

Hirota S, Isozaki K, Moriyama Y, Hashimoto K, et al. (1998). Gain-of-function mutations of c-kit in human gastrointestinal stromal tumors. Science 279: 577-580.

Jaiswal JK and Simon SM (2007). Imaging single events at the cell membrane. Nat. Chem. Biol. 3: 92-98.

Jaramillo S, Rios-Moreno MJ, Hernandez A, Amerigo J, et al. (2012). Gastrointestinal stromal tumors (GISTs): role of CD 117 and PDGFRA Golgi-like staining pattern in the recognition of mutational status. Rev. Esp. Enferm. Dig. 104: 128-133.

Joensuu H, Eriksson M, Sundby HK, Hartmann JT, et al. (2012). One vs three years of adjuvant imatinib for operable gastrointestinal stromal tumor: a randomized trial. JAMA 307: 1265-1272.

Li J, Gong JF, Li J, Gao J, et al. (2012). Efficacy of imatinib dose escalation in Chinese gastrointestinal stromal tumor patients. World J. Gastroenterol. 18: 698-703.

Li QS, Lee GY, Ong CN and Lim CT (2008). AFM indentation study of breast cancer cells. Biochem. Biophys. Res. Commun. 374: 609-613.

Lipman EA, Schuler B, Bakajin O and Eaton WA (2003). Single-molecule measurement of protein folding kinetics. Science 301: 1233-1235.

Miettinen M and Lasota J (2011). Histopathology of gastrointestinal stromal tumor. J. Surg. Oncol. 104: 865-873.

Miranda C, Nucifora M, Molinari F, Conca E, et al. (2012). KRAS and BRAF mutations predict primary resistance to imatinib in gastrointestinal stromal tumors. Clin. Cancer Res. 18: 1769-1776.

Mitchell W, Kaye P, Dhingsa R and Lobo D (2011). Education and imaging. Gastrointestinal: a gastrointestinal stromal tumour (GIST) arising in a Meckel's diverticulum. J. Gastroenterol. Hepatol. 26: 1812.

Pisters PW and Colombo C (2011). Adjuvant imatinib therapy for gastrointestinal stromal tumors. J. Surg. Oncol. 104: 896-900. 
Radmacher M (1997). Measuring the elastic properties of biological samples with the AFM. IEEE Eng. Med. Biol. Mag. 16: 47-57.

Rotsch C, Jacobson K and Radmacher M (1999). Dimensional and mechanical dynamics of active and stable edges in motile fibroblasts investigated by using atomic force microscopy. Proc. Natl. Acad. Sci. U. S. A. 96: 921-926.

Sihto H, Sarlomo-Rikala M, Tynninen O, Tanner M, et al. (2005). KIT and platelet-derived growth factor receptor alpha tyrosine kinase gene mutations and KIT amplifications in human solid tumors. J. Clin. Oncol. 23: 49-57.

Subramanian S, West RB, Corless CL, Ou W, et al. (2004). Gastrointestinal stromal tumors (GISTs) with KIT and PDGFRA mutations have distinct gene expression profiles. Oncogene 23: 7780-7790.

Svaldo LT, Cavalleri O, Krol S, Rolandi R, et al. (2006). Mechanical properties of single living cells encapsulated in polyelectrolyte matrixes. J. Biotechnol. 124: 723-731.

Vazquez J, Perez-Pena M, Gonzalez B and Sanchez A (2012). Gastrointestinal stromal tumor arising in the rectovaginal septum. J. Low Genit. Tract. Dis. 16: 158-161.

Wang D, Zhang Q, Blanke CD, Demetri GD, et al. (2012). Phase II trial of neoadjuvant/adjuvant imatinib mesylate for advanced primary and metastatic/recurrent operable gastrointestinal stromal tumors: long-term follow-up results of Radiation Therapy Oncology Group 0132. Ann. Surg. Oncol. 19: 1074-1080. 